\title{
Defects, faults and accidents of contemporary constructions
}

\author{
Michal Kraus ${ }^{1, *}$, Terezie Vondráčková ${ }^{1}$, and Vladimír Nývlt ${ }^{1}$ \\ ${ }^{1}$ Institute of Technology and Business in České Budějovice, Department of Civil Engineering, \\ 37001 České Budějovice, Czech Republic
}

\begin{abstract}
The paper deals with the defects, faults and accidents of contemporary constructions. The increasing number of constructions provides higher probability of occurrence of the defects. The defect is a lack of structure, which is caused by faulty design, inadequate or imperfect execution. The defect may affect the performance of the structure. The fault is the temporary or permanent loss of ability to perform its function. A defect may be causing disorder of structure. A special attention is paid to the defects and disruptions of buildings, which caused death or serious injuries (human or animal), safety hazards or major property damage. The aim of the paper is a description and analysis of data from the Construction and Technical Prevention System (CTPS) in the Czech Republic in several last years. It is necessary to take measures to prevent serious or excessively repetitive defects of buildings.
\end{abstract}

\section{Introduction}

Building production fluctuates in the Czech Republic over the last decade. Performance of construction industry increases up to 2008 , followed by a fall. Re-starting the construction production is evident since 2014. The construction sector is constantly expanding. The more buildings, the higher the probability of occurrence of a defect or fault is. The increasing number of buildings is not the only factor increasing probability of occurrence of defects. Building defects are not only a problem of old buildings. Also, new buildings can have defects [1]. The continuous monitoring and analysing of repeated and serious incidents is in the public interest for prosperity and development of society. Other important factors are quality of the realization, quality of used building materials, technical equipment, proper use and regular maintenance. Every human activity is encumbered with some risk and the unavoidable error rate. This risk represents risk of injury, damage, malfunction or accident. The buildings sector is no exception [2]. The risk analysis is a universal method for assessment of risk of defects, failures and collapses [3-5].

Defect can be defined as a lack of construction due to incorrect design, inadequate or imperfect execution decreasing performance of structures. The defect is not a change of construction compared to the original state. The defect of building can may have technical and factual consequences. The fault is temporary or permanent depletion capabilities of

\footnotetext{
* Corresponding author: info@krausmichal.cz
} 
construction. The fault is a change of construction compared to the original state. Faults may occur as a cause of the defect. Fault of construction has technical consequences. Accidents defines structural damage requiring repairs.

The Figure 1 shows the basic classification of the defects. In terms of function of construction, defects can be divided into serious and insignificant. The risk of injury, death or the emergence of substantial financial damage is characteristic of serious defects. According to the visibility, there are apparent defects and hidden defects. Apparent defect can be detected during routine maintenance or during the visual inspection. Hidden defect is not detectable by routine inspection or by maintenance [2].

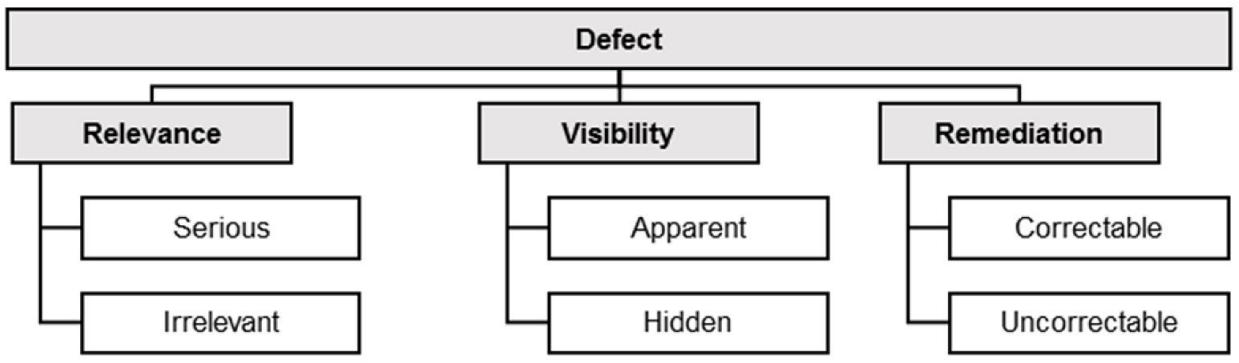

Fig. 1. Basic defect Classification [6].

\section{Source data and its structure}

The Construction and Technical Prevention System (CTPS) monitors serious or repeated defects in construction. The Institute for Spatial Development is responsible for the operation and administration of the system under the supervision of the Ministry of Regional Development of the Czech Republic. The CTPS is open access database. The system has been in operation since 2006. The Construction and Technical Prevention System monitors incidents that can be characterized by death of a person or animal, threat to life and health of people or animals, threat to the safety of the building and its equipment and by the emergence of property damage (approximately 18,000 Euros and more). According to the Building Act No. 183/2006 Sb., construction manager, construction supervision, developers and owners of buildings are obliged to notify the aforementioned incidents [6]. Other defects are not monitored by the system. The protocols have exactly defined form according to the Building Act. The structure of the analysed data is composed of year of occurrence, region, incidents, building category, functional unit/equipment and severity for each CTPS protocol.

Input data consist of a total of 516 reports recorded in the Construction and Technical Prevention System from 2010 to 2013. The sample includes the characteristic mix of contemporary buildings: residential, commercial, agricultural, industrial, educational, religious, government, transport building and infrastructure, site facilities and others from all regions of the Czech Republic. Overall, incidents are divided into nine categories of buildings.

In the context of the examined data sample, the statistical character "incident" represents situation that lead to defects, injuries or accidents in the construction practice. Generally, it is defined 6 kinds of incidents:

- Carbon monoxide poisoning, due to gas heaters, boilers and karma. In some cases, intoxication ends with the death of a person.

- Explosion, which was due to poor indoor installation or as yet unidentified causes. In most cases, it is the explosion of a gas pipeline as indoor installation of buildings.

- Fall from height, caving or injured animal. 
- Fall from height, caving or personal injury.

- Fire, usually caused by careless use of open fire, the failure of indoor installation of building or by lightning. This category does not include the fire, which was created after the explosion.

- Structural collapse, building defect without explosion or fire leading to collapse or damage to construction.

Statistical sign "functional unit/equipment" describes the part of the structure characterized by a primary defect. The severity of the incidents is divided into five degrees from large financial damage without injury to the person's death (defined below).

\section{Results}

The sample is subjected to basic exploratory analysis. Absolute frequencies and relative frequencies are monitored for each group incidents. Incidents are analysed according to the territory, the building categories, and the functional unit of the building and according to the degree of severity.

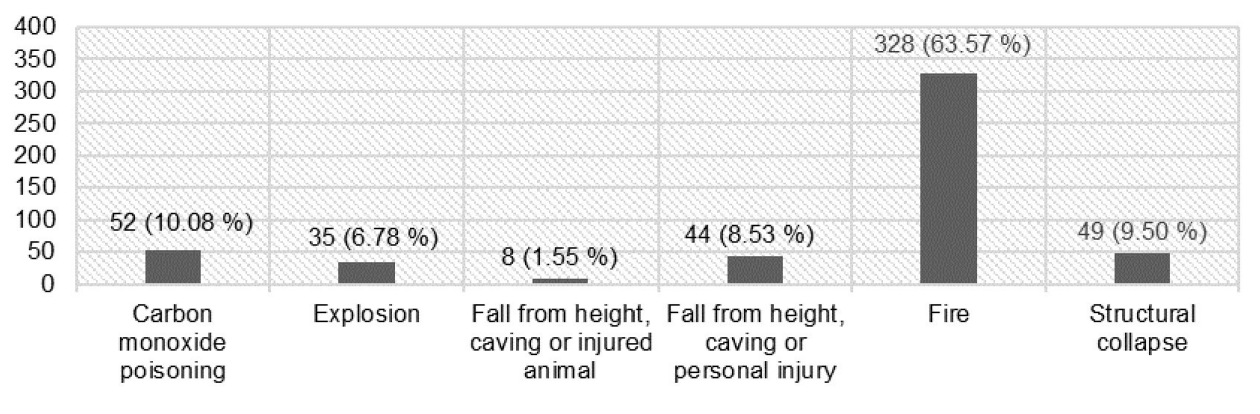

Absolute frequency (Relative frequency)

Fig. 2. Total overview of incidents.

The most common incident (modus) is a fire. It covers more than $64 \%$ of the sample. The main causes of fires are negligent conduct of building users as well as technical faults of functional units of constructions and building equipment. Relatively significant proportion also has carbon dioxide poisoning (10.08\%), personal injuries $(8.53 \%)$ and collapsed of structures $(9.50 \%)$. Fall from height and fall into the ditch is the most common cause of injury. Vice versa, injuries to the animals has the smallest representation in data sample.

\subsection{Territorial representations of incidents}

The sample includes incidents from all 14 regions of the Czech Republic. As it is shown in Figure 3, the territorial representation of the incidents gives the total distribution of defects, faults and accidents in the Czech Republic. The most common area of monitored incidents is evident in region of Moravia-Silesia. Almost 16\% of monitored incidents occurred in the Moravia-Silesia Region. The lowest density of incidents is region of Liberec (only 3.30\%). It is necessary to look at the statistics territorial representation of incidents with considerable reserve due to a difference in the density of population and development in various regions. 


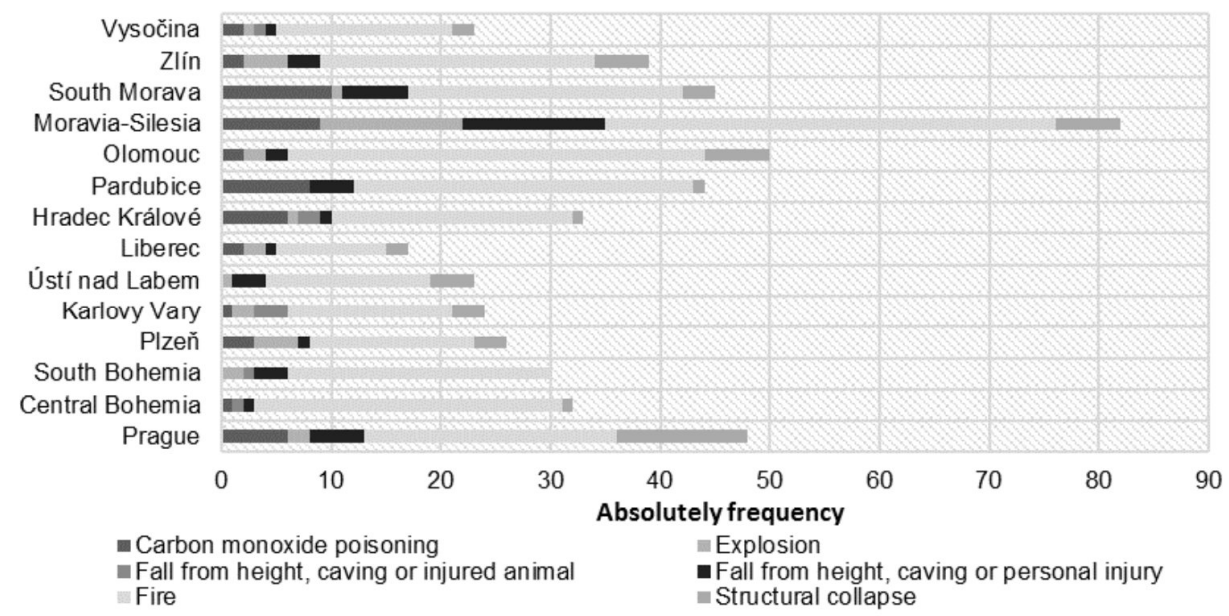

Fig. 3. Overview of incidents by regions in the Czech Republic.

\subsection{Building categories of incidents}

Building defects and incidents are common problems in any built structures. Altogether is defined nine categories of buildings (Figure 4). It is clear, the number of fires is the greatest in all categories of buildings. In the context of the occurrence of fires, residential buildings are most at risk. More than $55 \%$ of all fires are monitored in residential buildings. The buildings and storage are the second highest risk category. Conversely, the smallest number of incidents is monitored for educational, religious and government buildings. This differentiation confirms the diversity of fire protection requirements of various categories of buildings.

Site facilities are statistically significant for the high proportion of endangering the health and lives of people. The prevention and strict adherence to principles in the field of safety and health at work is an integral prerequisite for reducing the number of serious incidents in the future.

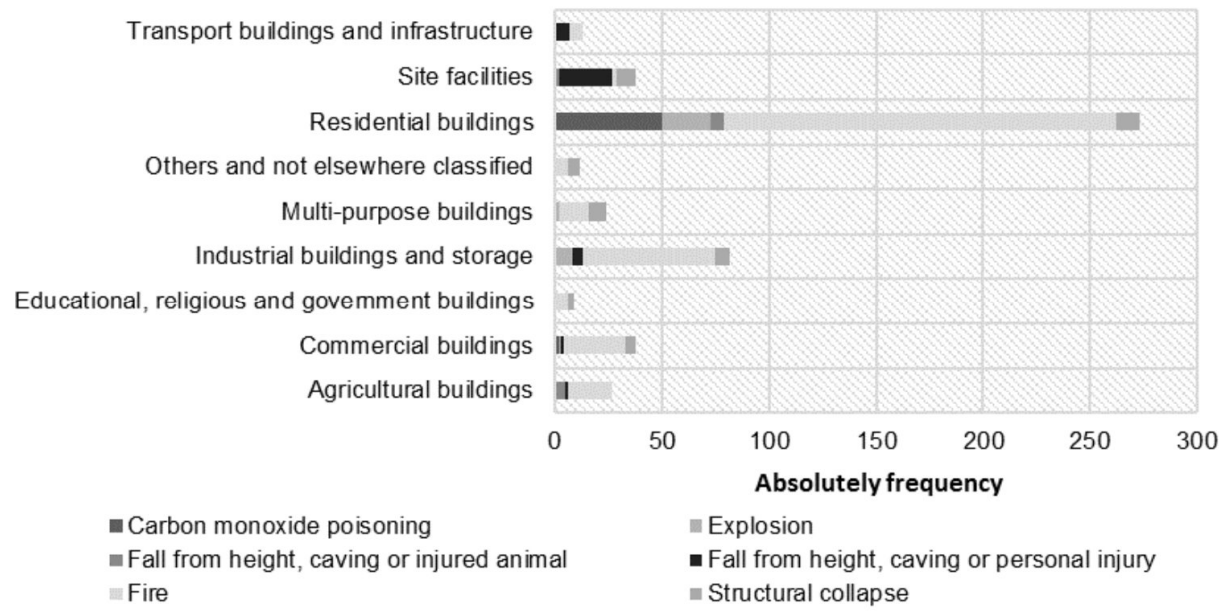

Fig. 4. Overview of incidents by building categories. 


\subsection{Functional unit and equipment of incidents}

It is determined a total of 15 kinds of functional units or equipment (A-O) based on input data. Definition of functional units and their occurrence (relative frequencies) is described in Figure 4. Almost half of the data file (45.16\%) consists of incidents caused by a combination of functional units and internal equipment as buildings. A significant proportion of incidents have also the roof structures $(16.86 \%)$ and the heating systems $(12.40 \%)$. Other functional units, equipment and indoor installations are equally risky for occurrence of defects and the threat to lives and health.

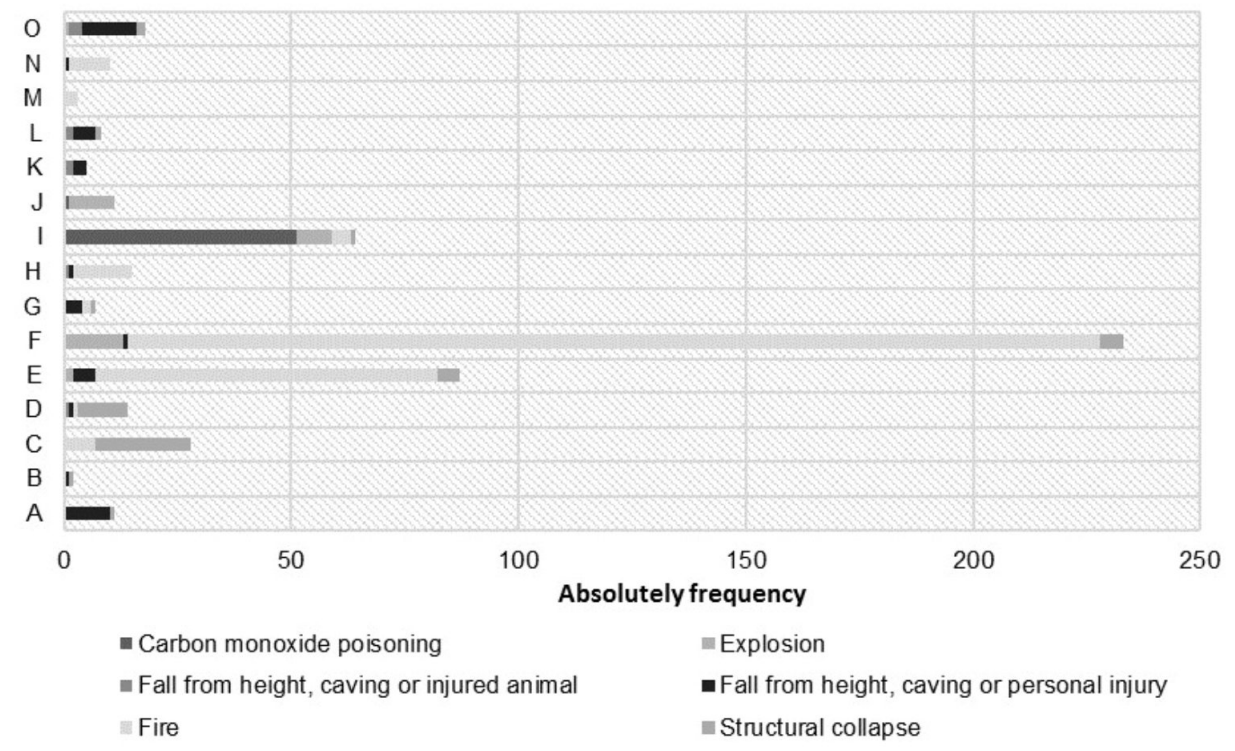

Legend:

\begin{tabular}{|c|l|c|}
\hline Symbol & Functional unit/Equipment & Relatively frequency \\
\hline A & Excavation work & $2.13 \%$ \\
\hline B & Foundation structures & $0.39 \%$ \\
\hline C & Walls and partitions & $5.43 \%$ \\
\hline D & Ceilings and horizontal structures & $2.71 \%$ \\
\hline E & Attic and roof & $16.86 \%$ \\
\hline F & Combination of constructions and interior equipment & $45.16 \%$ \\
\hline G & Transportation equipment & $1.36 \%$ \\
\hline H & Technological equipment and interior equipment & $2.91 \%$ \\
\hline I & Heating (indoor installation of buildings) & $12.40 \%$ \\
\hline J & Pipeline (indoor installation of buildings) & $2.13 \%$ \\
\hline K & Sewerage (indoor installation of buildings) & $0.97 \%$ \\
\hline L & Water supply (indoor installation of buildings) & $1.55 \%$ \\
\hline M & Ventilation (indoor installation of buildings) & $0.58 \%$ \\
\hline N & Electrical installation (indoor installation of buildings) & $1.94 \%$ \\
\hline O & Others and not elsewhere classified & $3.49 \%$ \\
\hline
\end{tabular}

Fig. 5. Overview of incidents by functional units/equipment. 


\subsection{Severity of incidents}

The input data include the severity of the incident. It is stated 5 degrees of severity from minor to serious. The first degree of severity (1) includes only significant financial loss without endangering of humans and animals. As mentioned above, the financial damage must be more than 18000 Euros. Incidents with less financial damage are not analysed. The second degree of severity involves incidents threatening the lives and health of animals. The third degree of severity is defined by the deaths of animals. The fourth degree of severity involves the incidents of threats to human life and its health. The fifth degree (5) of severity, the most serious, represents the situations ending with death of one or more persons.

Figure 5 shows the final matrix of incidents and degrees of severity. Nearly $45 \%$ of all serious incidents end with serious financial damage. The rest defines cases of injury or death to persons or animals. More than $35 \%$ of incident represents injured people and $16 \%$ of incidents ends by death. In terms of fatal accidents, the fire (43\%) and fall from height or caving (21\%) are the major cause of death. In the context of the categories of buildings, the residential buildings predominate with a share of $55 \%$.

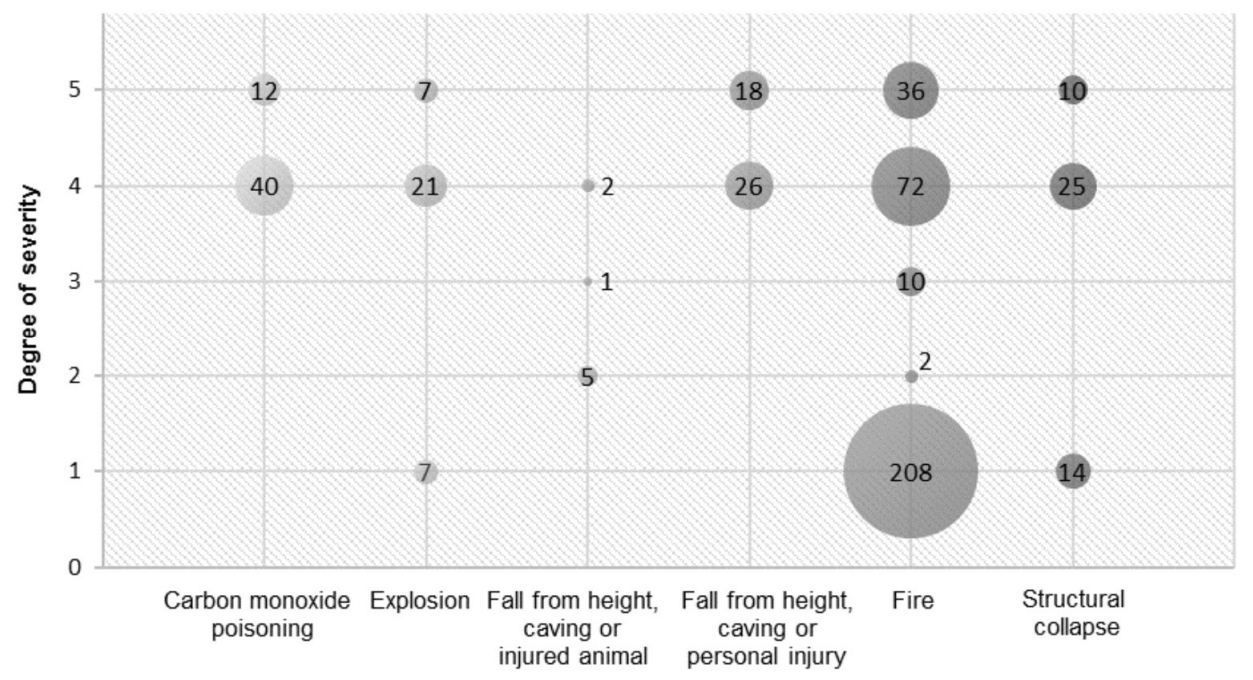

Fig. 6. Matrix of incidents and degrees of degrees of severity.

\section{Conclusions}

The aim of the paper is to evaluate and exploratory analysis of serious and recurring incidents of contemporary constructions. The analysis of incidents of contemporary construction brings new knowledge. More than half of the incidents the fire. It is necessary to pay attention to fire precautions and prevention not only during the construction phase and use, but also in the design phase. Negligence is a major cause of fires. The negligent conduct includes smoking, incorrect servicing of heaters and neglect of safety regulations. It is necessary to observe the principle of working with open flame, regular maintenance of technical equipment of buildings in order to minimize fires. 


\section{References}

1. N.N.O. Bakrim M.A.O. Mydin, European Journal of Technology and Design 3 (2014)

2. K. Kubečka, Transactions of the VŠB - Technical University of Ostrava, Civil Engineering Series, 1, 27 (2010)

3. K. Kubečka, P. Vlček, D. Kubečková, AMR 899, 535-538 (2014)

4. K. Kubečka, P. Vlček, D. Kubečková, D. Pieszka, AMR 1020, 751-755 (2014)

5. M. Kraus, P. Bednářová, K. Kubečka AMM, 824 657-665 (2016)

6. Act No. 183/2006 Coll., on town planning and the building code (the Building Act) (2006) 